\title{
Fatigue Life Prediction of Long-Life Pavement Using Frequency-Domain Analysis
}

\author{
Wei Baoli, Guo Chengchao, Cui Can
}

Institute of Water Conservancy and Environment, Zhengzhou University, Zhengzhou 450001, China

\begin{abstract}
This paper proposes and analyzes a pavement fatigue life prediction method for asphalt and cement concrete pavements. A theoretical frequency domain analysis involving pavement fatigue life was conducted. By using the vehicle-road coupled vibration system model, the random vibration between a vehicle and road caused by road roughness was analyzed. In this paper, it is proposed that the random power spectral density of the vehicle-road coupled vibration is a useful tool to predict the fatigue life of pavements, and the corresponding prediction procedures are proposed, aimed at providing some reference for long-life pavement design and performance evaluation.
\end{abstract}

Keywords: long-life pavement; fatigue life prediction; random vibration; power spectral density

\section{Introduction}

Long-life pavement (LLP) is a new trend in development. In recent years, long-life pavement research has become globally popular. LLP refers to the design of pavement life of longer than 40 years. Compared to the existing pavement system, daily maintenance of this new pavement structure requires lower total cost [1].

In the 1890 s, European scholars proposed the concept of long-life pavement, with the first application attempted by the British [2], and it was then applied and developed in Japan. Japanese researchers widely believe that the performance of long-life pavement can more than double the conventional pavement performance, where the average expectancy of this pavement structure is approximately 50 years, and refer to it as long-service pavement (LSP) [3].

The structure of LLP [4], as considered by the general academic researcher, generally consists of several functional layers. (1) High stress region is located 100-150 mm under the wheel load. In this region, rutting, cracking, and other damage occurs often. (2) Good performance area, which consists of asphalt concrete, is located $40-75 \mathrm{~mm}$ under the surface layer. The texture depth of this layer should meet the requirements for vehicle driving, and should have good resistance to rutting. (3) The middle stress dispersion layer is composed of asphalt concrete. This layer generally has a thickness of 100-175 mm. Its function is connection and transition. In addition, the load is spread in the region, because of the high stiffness of asphalt concrete, which contributes an anti-rutting function. (4) Fatigue dissipation layer is located $75-100 \mathrm{~mm}$ under the hot mix asphalt (HMA) mixture base. This area is greatly influenced by fatigue. To reduce the effects of fatigue, the function layer generally consists of low-stiffness, waterproof, and fatigueresistant asphalt concrete. (5) The sensitive damage layer is commonly located at the bottom of the HMA base. The region experiences maximum tensile strain, and is most vulnerable to fatigue damage. To prevent pavement structural damage and control asphalt layer bottom-up fatigue, it is necessary to focus on controlling the bending tensile strain in this region.

In 2005, the Key Technology Research on Long-Life Asphalt Pavement Overloaded Traffic initiative, presided over by Academician Sha Qinglin and undertaken by Changsha Uni-

Received date: November 18, 2017; Revised date: November 30, 2017

Corresponding author: Guo Chengchao, Institute of Water Conservancy and Environment, Zhengzhou University, Associate Professor. Major research field is theory and technology of infrastructure testing and restoration. E-mail: wangchench@163.com

Funding program: CAE Advisory Project “Strategic Studies on Safety of Major Structures of Transportation Infrastructure” (2015-XZ-28)

Chinese version: Strategic Study of CAE 2017, 19(6): 080-085

Cited item: Guo Chengchao et al. Fatigue Life Prediction of Long-Life Pavement Using Frequency-Domain Analysis. Strategic Study of CAE, https://doi.org/10.15302/ J-SSCAE-2017.06.012 
versity of Science and Technology, was listed in the west traffic construction science and technology projects considered of vital significance to China. In October 2005, "long-life pavement structure" was listed in the first place of the national top 10 scientific research projects established by the national ministry of transport. Professor Wang Xuancang of Chang'an University, proposed a long-life pavement structure with the characteristic of "couple hardness with softness, to complement each other's advantages", which has been widely adopted in engineering.

Currently, global academia and scientists recognize that longlife pavement's design is 40 years or more. During the design life, the pavement structure generally can ensure utilization, and major damage will not occur. The normal damage to pavement surface will be regularly maintained. The need to overhaul the main load-bearing layer generally does not occur or rarely appears. During the whole life cycle, the construction cost (because of the thick pavement layer) may be higher, but the maintenance costs are lower, therefore, the overall cost is the most economic.

\section{Pavement fatigue life}

Pavement fatigue means that the internal performance of the material becomes worse under repeated loads (generally smaller than static loads). Fatigue life is the span from its beginning to the time when fractures occur under load, or the cycle times of the stress or strain experienced before fatigue failure [5-8].

Under repeated load and environmental conditions (mainly water and temperature), the pavement wear-resistance, anti-aging, water damage-resistance, fatigue-resistance, rutting-resistance, and temperature shrinkage crack-resistance constantly decline. Correspondingly, pavement surface roughness, comfortable performance (surface function), noise-resistance, slip resistance, and so on also deteriorate, and the pavement structure becomes susceptible to fatigue damage.

\subsection{Fatigue life of asphalt concrete pavement}

In 1942, Porter observed the phenomenon of fatigue damage, and found that the wheel load cycles of only a few million times were necessary before pavement damage occurred at low deflection (less than $0.5-0.75 \mathrm{~mm}$ ). In the $1950 \mathrm{~s}$, Nijboer et al. pointed out that cracks were the result of fatigue, which depended on deflection and repeated load cycles, and then highlighted how asphalt concrete cracks occur when the flexural-tensile stress exceeds the material's allowable strength in late life $[9,10]$ Chinese scholars began to systematically research on pavement fatigue properties in the 1960s. In theory and practice, pavement fatigue cracks appear under the coupling of tensile, compressive, and shear stress caused by moving wheel repeated loads. The cracks gradually expand, form crack coalescence, and cause fatigue damage. The fatigue failure mechanism can be explained by fracture mechanics theory.
Research on the fatigue characteristics of asphalt pavement mainly focus on the following two aspects: fatigue test [10] and mechanical analysis [11]. The stress-strain curve is obtained by the fatigue test, and the fatigue characteristics are summarized using the constitutive relationships. The mechanical analysis principle is used to study the process of crack formation, the mechanical law associated with the crack is found, and the fatigue life of the material is predicted [12].

Britain's Brown et al. [13] studied fatigue properties of an asphalt mixture on pavement structure and conducted indoor fatigue tests to obtain the fatigue equation of the asphalt mixture and used the equation in the British pavement design code.

Monismith et al. [14] (University of California at Berkeley in the United States) focused on the fatigue test of asphalt mixtures, mainly involving laboratory bending fatigue tests. He demonstrated regularity of cracks in the material and verified the formula developed through a full-scale outdoor test. Asphalt fatigue equation applied by the American Asphalt Institute (AI) for asphalt pavement design is the fatigue law developed by Monismith.

The asphalt mixture fatigue equation of AI was improved by the American Association of State Highway and Transportation Officials (AASHTO) in its structure design guidelines in 2002. The improved asphalt mixture fatigue equation considers air voids, asphalt content, dynamic modulus, and other properties [15].

Since 1960, researchers at SHELL Oil Company have performed research on bituminous mixtures, and in 1981 revised their standard fatigue equation to share the same expression method with the United States specification and the British specification [16].

\subsection{Fatigue life of cement concrete pavement}

Cement concrete pavement fatigue life is an important indicator of the Chinese cement concrete pavement structure design method. In China the fatigue fracture caused by the combined effect of vehicle load and the temperature has been the dominant design indicator. On the premise of meeting the performance requirements of the pavement structure, the number of repeated loads has been defined as the fatigue life when fatigue damage occurs in concrete pavement. Therefore, the prediction of the fatigue life of cement concrete is also significant.

In recent years, with the increasing attention on concrete structure and enhancing its performance, the high stress area often appears in the structure during its life and there will be frequent alternation of tension and compression that lead to concrete fatigue problems. Therefore, the fatigue problem of concrete materials has become of concern to researchers. Since then, with the improvement of experimental conditions, increasing numbers of research results have emerged, which have also promoted the vigorous research of concrete fatigue. 
Research for cement concrete pavement fatigue life forecasting methods mainly fall into two major categories: laboratory tests and field tests. Owing to the limitations of field testing, Chinese researchers mainly adopt the laboratory test method and utilize the bending fatigue test of the concrete specimen to evaluate the relationship between repeated stresses and remaining service life based on fatigue, considering the relevant influence factors. Through these, they consider that the occurrence of fatigue is a gradually forming process leading to failure. This law can be characterized by the test data, and is used to determine the concrete fatigue life prediction formula. The main problem with the laboratory test method is that many repetitions of load on the material are required, and it is difficult to carry out this type of analysis when the funds are insufficient. Furthermore, the empirical formula obtained by this method can only be used under the condition that the concrete pavement has no initial defects, and its scope of use is limited in real-world engineering practice. The field experiment method is mainly used by European and American researchers using test roads, among which the test road of the American Association of State Highway Officials (AASHO) is the most typical test road. This method mainly simulates the fatigue failure of pavement by using accelerated pavement tests on a full-scale test road outdoors, and determines the fatigue characteristics of the cement concrete pavement to predict fatigue life.

\section{Frequency domain analysis method for fatigue life prediction}

The application of fatigue life prediction in the field of civil engineering was adopted by civil engineers for life prediction in the field based on mechanical engineering principles. The main methods of fatigue life prediction fall into two major categories: time domain methods and frequency domain methods. The frequency domain method estimates the fatigue life of parts by using frequency-related structural parameters. Among these structural parameters, the stress power spectral density (PSD) is the most commonly used parameter. There are few unknown quantities involved in predicting the fatigue life in the frequency domain, which is convenient for engineers to use and is widely recognized. The frequency domain method can be categorized as four types, discussed below.

\subsection{Narrow-band distribution method (Bendat method)}

The narrow-band distribution method was first proposed by Bendat et al. [17]. This method estimates fatigue life by using the stress power spectral density. Bendat et al. [17] found that the peak probability density function of the random signal decreases gradually over the bandwidth, and can be expressed by the Rayleigh function. In addition, the number of peaks and troughs is the same as the narrow-band characteristics of random signals, and the probability density functions of signals can also be expressed as Rayleigh functions. According to the Miner linear cumulative damage theory, the fatigue damage of the structure can be expressed as a continuous distribution function of fatigue damage related to the stress state in time $T$. In general, the fatigue life curve in engineering can be used to describe the fatigue properties of the material. When a random signal exists in a narrow band and the number of stress cycles per unit time is equal to the mean positive crossing rate. The fatigue damage result can thus be calculated in the narrow band condition and the failure time of life and the lifetime of a component before failure can be predicted.

\subsection{Wirsching-Light method}

Due to the great difference between the peak value expectation rate and the average crossing rate in the unit of time for broadband process, the life estimated by the narrow-band distribution method will include a very large error. Thus, for the broadband random process, if the narrow-band distribution method is to be used, it must be modified. Wirsching et al. [18] modified the formula based on the power spectral density, and used the modified formula to predict lifetime for wide-band random vibration.

\subsection{Tovo-Benasciutti method}

The probability density function of the rain flow amplitude proposed by Tovo and Benasciutti is also a correction method based on the narrow band model. By using fatigue damage and a fatigue life curve, the result for fatigue cumulative damage can be obtained to calculate the remaining time of fatigue life [19].

\subsection{Dirlik rain-flow amplitude distribution model}

By analyzing the power spectral density function and using 70 data samples, Dirlik [20] generated 70 types of waveforms in the time domain by Monte Carlo simulation. He estimated the probability density function of the rain-flow circulation amplitude by using an empirical expression. Dirlik pointed out that the distribution function of the cyclic amplitude of rain flow is a compound function of the Rayleigh distribution function and the exponential function by deriving the empirical formula. The results of fatigue cumulative damage can be obtained by combining the empirical value of the formula with the fatigue damage and fatigue life curve, and then the residual time of fatigue life can be calculated.

\section{Power spectrum method for prediction of fatigue life of pavement}

Because of the frequent occurrence of high, light, and large 
modern engineering structures, structural fatigue damage has become one of the main causes of economic loss. The generalized fatigue load not only refers to the cyclic loading, but also includes the random fatigue load. The fatigue of materials under cyclic loading is a conventional problem, while the random characteristics of fatigue load remain unclear at present. However, in the field of engineering, the random vibration characteristics of a structure are often prominent, and the random fatigue of the structure often leads to serious failure of the structure.

The interaction of vehicles and roads involves two research areas or two systems (vehicles and roads). For a vehicle-road coupled system, the generation of its random excitation is related to the pavement roughness, that is to say, the research of a vehicle-road coupled system should be placed in the theoretical category of random vibration.

\subsection{Power spectrum of pavement structure}

The vibration of the vehicle-road coupled system is related to the pavement elevation, and the pavement elevation can be characterized by its roughness. To analyze the random vibration characteristics of a vehicle-road coupling system, pavement roughness is a factor that must be considered. In other words, pavement roughness is an excitation source and has certain randomness.

According to the standard of GB/T 7031-2005 Mechanical Vibration-Road Surface Profiles-Reporting of Measured Data, it is recommended to describe the statistical characteristics of pavement by the power spectral density function of pavement by different grades. The power spectrum of pavement roughness can be fitted by Eq. (1).

$$
G_{d}(n)=G_{d}\left(n_{0}\right)\left(\frac{n}{n_{0}}\right)^{-\beta}
$$

where $n$ is the spatial frequency $\left(\mathrm{m}^{-1}\right)$, representing the number of wavelengths appearing in the length of each meter and is the reciprocal of the wavelength. The value of $n$ for the pavement power spectrum is $n \in(0.011,2.83) . n_{0}$ is a reference spatial frequency $\left(\mathrm{m}^{-1}\right)$ with a value of $0.1 . G_{d}\left(n_{0}\right)$ is the pavement roughness coefficient $\left(\mathrm{m}^{3}\right)$, and is the power density function value of the pavement related to the pavement grade under the reference spatial frequency $n_{0} . \beta$ is the frequency index, which corresponds to the slope of the double logarithmic coordinate line and determines the frequency structure of the power spectral density function of the pavement.

The GB/T 7031-2005 standard divides the common road into a total of eight levels A-H according to the pavement roughness coefficient, and provides the geometric mean value of $G_{d}\left(n_{0}\right)$. According to the statistics, the power spectrum of high grade highway pavement structure in China is basically in the A-C level, and most highways are concentrated in the two grades of B and C. Eq. (1) represents the statistical characteristics of the power spectrum of the spatial frequency, but in practice, the influence of the road excitation frequency is not only the pavement roughness, but also the speed of the vehicle. When the driving speed increases, the excitation frequency increases; otherwise the excitation frequency decreases. To consider the effect of vehicle speed $v$, the time frequency $f$ and angular spatial frequency $\omega$ are used to replace the spatial frequency $n$.

$$
\begin{gathered}
f=v n \\
\omega=2 \pi f=2 \pi v n \\
G_{n}(f)=\frac{G_{n}(n)}{v}
\end{gathered}
$$

and substituting Eqs. (2)- (4) into Eq. (1), we obtain Eq. (5) and Eq. (6) as follows, where the frequency index $\beta$ of the fitting power spectral density equals 2 .

$$
\begin{gathered}
G_{n}(f)=\frac{G_{n}\left(n_{0}\right)}{v} \cdot\left(f / v n_{0}\right)^{-2} \\
G_{n}(\omega)=4 \pi^{2} \frac{G_{n}\left(n_{0}\right)}{v} \cdot\left(\omega / v n_{0}\right)^{-2}
\end{gathered}
$$

Eq. (6) is further simplified to the following:

$$
G_{n}(\omega)=4 \pi^{2} G_{n}\left(n_{0}\right) \frac{v n_{0}^{2}}{\omega^{2}}
$$

in Eq. (7), when $\omega$ tends to $0, G_{n}(\omega)$ is infinite. To avoid this situation, the lower cut-off angle frequency $\left(\omega_{00}\right)[21]$ is introduced in practical application. Substituting $\omega_{00}=2 \pi v n_{00}$ into Eq. (7), we obtain:

$$
G_{n}(\omega)=4 \pi^{2} G_{n}\left(n_{0}\right) \frac{v n_{0}^{2}}{\omega_{00}^{2}+\omega^{2}}
$$

if the Gaussian white noise input is $W$, where $G_{W}(\omega)=1$, then if both sides of Eq. (8) are multiplied by $G_{W}(\omega)$, Eq. (9) can be obtained if the value of 1 is applied to the left side of Eq. (8) and the Gaussian spectrum term is applied to the right hand side:

$$
G_{n}(\omega)=4 \pi^{2} G_{n}\left(n_{0}\right) \frac{v n_{0}^{2}}{\omega_{00}^{2}+\omega^{2}} \cdot G_{W}(\omega)
$$

according to the theory of random vibration, the frequency response function $H(\omega)$ of Eq. (9) can be obtained:

$$
H(\omega)=\frac{2 \pi n_{0} \sqrt{G_{n}\left(n_{0}\right) v}}{\omega_{00}+j \omega}
$$

then the differential equation in the time domain can be obtained:

$$
\dot{q}(t)=-2 \pi n_{00} v q(t)+2 \pi n_{0} \sqrt{G_{n}\left(n_{0}\right) v} \cdot w(t)
$$

where $\omega(t)$ is a white noise signal with a power spectrum of 1 . $n_{00}$ is the lower cut-off space frequency, and the GB/T 7031-2005 standard recommended $n_{00}$ is $0.011 \mathrm{~m}^{-1} . G_{n}\left(n_{0}\right)$ is the pavement 
roughness coefficient corresponding to the road level and can take the geometric mean of pavement roughness recommended by GB/T 7031-2005. In Eq. (11), $v$ is the vehicle speed $(\mathrm{m} / \mathrm{s})$, and $q(t)$ is the road surface random elevation $(\mathrm{m})$, that is, the pavement roughness.

\subsection{Prediction of pavement fatigue life measured by power spectrum}

It has usually been considered that in the traditional vibration model, the road deformation caused by vehicles can be neglected compared with the pavement roughness. Therefore, the traditional linear model assumes that the road surface is rigid, that is, the road system is not involved in vibration. The dynamic load of the vehicle is obtained by the random vibration of the vehicle, and then the dynamic response of the road is studied by using the dynamic load of the vehicle. The model does not consider the influence of vehicle-road system coupled vibration and is not completely applicable in the case of increasing vehicle load and higher speed. Under the random vibration problem of vehicle-road system coupling, the frequency and amplitude of the vehicle-road system will change. In view of the above issue, based on the traditional model research, a linear model of random vibration of a vehicle-road coupled system is established by the author of this article. Random vibration theory is used to solve the random vibration response of the vehicle-road coupled system by applying frequency domain analysis.

According to the theory of random vibration, if the spectral density function $S(\omega)$ of the pavement roughness is given, the following equation applies to the displacement spectral density function $S_{z}(\omega)$ of the response.

$$
S_{z}(\omega)=\left|H_{z}(\omega)\right|^{2} S(\omega)
$$

and simultaneously:

$$
\begin{aligned}
& S_{z}(\omega)=\omega^{2} S_{z}(\omega) \\
& S_{z}(\omega)=\omega^{4} S_{z}(\omega)
\end{aligned}
$$

where $H_{z}(\omega)$ is the frequency response function; $S_{z}(\omega)$ is the velocity spectral density function of the response, and $S_{\bar{z}}(\omega)$ is the acceleration spectral density function of the response.

The displacement spectrum density, the velocity spectrum density, and the acceleration spectrum density can be subdivided into a vehicle-road interaction equation, and the load power spectral density $S_{F}(\omega)$ can be obtained. $S_{F}(\omega)$ is input into the vibration equation of the pavement structure in the form of PSD load. By using $H_{Z}(\omega)$ (the frequency response function), the relationship between the input load and the output stress at a specific location of the structure (the pavement surface and the base top surface, respectively) can be obtained. $G(f)$ is used to represent the stress PSD of the damaged structural part. Then

$$
G(f)=|H(f)|^{2} \cdot W(f)
$$

where $H(f)$ is the transfer function of a random vibration system and $W(f)$ is the power spectral density function of the input load of the system. The root mean square value of the stress can be obtained by the following equation.

$$
\sigma^{2}=\int_{-\infty}^{+\infty} G(f) \mathrm{d} f
$$

After obtaining the stress amplitude probability density function of the system response, the fatigue life of the structure can be further estimated based on the relationship between the stress level of the pavement structure material and the material fatigue life (S-N curve), and applying the linear Palmgren-Miner damage theory.

\section{Conclusions}

From the discussion of the prediction method of fatigue life of long life pavement, the main conclusions are as follows.

(1) The development process of long-life pavement is expounded. The fatigue life equation of long-life pavement according to pavement structure material classification is described. The method and steps of fatigue life prediction for long-life pavement are clarified. Combined with vehicle-road coupled vibration theory, the composition and reconstruction process of the pavement power spectrum are analyzed, and the roughness function of different pavement grades is constructed by using the power spectrum fitting function and the corresponding road power spectrum. The applicable reconstruction algorithm is also provided.

(2) It is pointed out that vehicle-road coupled vibration is a random vibration process based on probability statistics theory, and the fatigue life of pavement can be predicted through frequency domain analysis. By using the theory of random vibration, the equation and prediction procedure of fatigue life prediction are given by using the power spectral density function of the vibration response of the pavement structure. The method proposed by the author can be used as a method for pavement life prediction, and can be used for reference in the design and evaluation of long-life pavement.

Although the fatigue life prediction based on the frequency domain method is analyzed for the road, the accuracy and reliability of a structure's random fatigue life prediction based on this method requires further verification. With additional research under specific conditions, the various material S-N curves will be more meaningful for real-world design.

\section{References}

[1] Yi X Y. Discussion and application of long-life flexible pavement technology [J]. Journal of Highway and Transportation Research and Development, 2015, 32(6): 25-31. Chinese.

[2] Song B, Xue Z J, Zhou X L, et al. Research summary of Occident long-life asphalt pavement on existing pavement [J]. Municipal Engineering Technology, 2016, 34(1): 17-22. Chinese.

[3] Feng Z A, Wang X C, Li G S. Study and design and construction 
technology on the typical structure of long-life pavement [M]. Beijing: China Communications Press, 2007. Chinese.

[4] Ma S J. Application and development of long-life asphalt pavement in Shandong [J]. China Highway, 2016(7): 75-75. Chinese.

[5] Hu P, Wu Q, Pan X D. Test and feasibility of wheel track weight distribution and effect on the fatigue life of asphalt concrete pavement [J]. Highway, 2015(2): 20-24. Chinese.

[6] Lu J W, Wang X Z, Wu W. Fatigue reliability analysis on the driving axle housing of a light truck under random road excitation $[\mathrm{J}]$. Automotive Engineering, 2016, 38(1): 122-126. Chinese.

[7] Sun C. The analysis of long life asphalt pavement fatigue model and design index (Master's thesis) [D]. Harbin: Harbin Institute of Technology, 2015. Chinese.

[8] Bateman D, Carswell I, Caudwell L, et al. Practical verification of the theory behind long-life asphalt pavements [C]. Euro-asphalt \& Euro-bitumen Congress, 2016.

[9] Gao J H. Study on the modulus of pavement structure of asphalt pavement layer based on fatigue life [J]. Journal of China \& Foreign Highway, 2013, 33(4): 109-113. Chinese.

[10] Zhang D L. Asphalt pavement engineering handbook [M]. Beijing: China Communications Press, 2003. Chinese.

[11] Cong Z M, Zou X L, Gong H R. Influence of heavy load on rutting and fatigue life of asphalt pavement [J]. Journal of Wuhan University of Technology, 2013, 35(4): 54-58. Chinese.

[12] Shen J A. Foreign design method of asphalt pavement [M]. Beijing: China Communications Press, 2004. Chinese.
[13] Brown S F, Brodrick B V, Tekieli K, et al. A torsional hollow cylinder research apparatus for studying the permanent deformation characteristics of asphalt [J]. Road Materials \& Pavement Design, 2013, 14(sup1): 65-85.

[14] Monismith C L, Long F. Overlay design for cracked and seated Portland cement concrete (PCC) pavement-Interstate route 710[R]. USA: University of California-Berkeley, 1999.

[15] AASHTO. Guide for mechanistic-empirical design of new and rehabilitated pavement structures final report [R]. Washington D C: National Cooperative Highway Research Program, 2004.

[16] Shell International Petroleum Co. Ltd. Shell pavement design manual-asphalt pavement and overlays for road traffic [M]. London: Shell International Petroleum Co. Ltd., 1981.

[17] Bendat J S, Piersol A G. Measurement and analysis of random data [M]. New York: John Wiley and Sons, 1966.

[18] Wirsching P H, Torng T Y, Martin W S. Advanced fatigue reliability analysis [J]. International Journal of Fatigue, 1991, 13(5): 389-394

[19] Benasciutti D, Tovo R. Comparison of spectral methods for fatigue analysis of broad-band Gaussian random processes [J]. Probabilistic Engineering Mechanics, 2006(21): 287-299.

[20] Dirlik T. Application of computers in fatigue analysis (Doctoral dissertation) [D]. Coventry: University of Warwick, 1985.

[21] Yu F, Lin Y. Automobile system dynamics [M]. Beijing: China Machine Press, 2005. Chinese. 